\title{
INFLUÊNCIA DO GRAU DE INSUFICIÊNCIA HEPÁTICA E DO ÍNDICE DE CONGESTÃO PORTAL NA RECIDIVA HEMORRÁGICA DE CIRRÓTICOS SUBMETIDOS A CIRURGIA DE TEIXEIRA-WARREN
}

\author{
Fabio Gonçalves FERREIRA ${ }^{1}$, João Ricardo DUDA', Márcia OLANDOSKI² e Armando De CAPUA Jr. ${ }^{1}$
}

\begin{abstract}
RESUMO - Racional - A hipertensão portal com sua principal complicação, a hemorragia digestiva alta varicosa, são importantes causas de morbimortalidade em cirróticos. A cirurgia de Teixeira-Warren é uma derivação portal seletiva, adotada em doentes Child-Pugh A e B para tratamento da hemorragia varicosa por hipertensão portal não responsiva à terapêutica clínico-endoscópica após o quadro agudo. O índice de congestão portal baseia-se em valores obtidos pela ultra-sonografia Doppler abdominal e encontra-se elevado em pacientes com hipertensão portal. Objetivo - Verificar se o índice de congestão portal e o grau de insuficiência hepática (Child-Pugh) são fatores preditivos de recidiva hemorrágica após a cirurgia de Teixeira-Warren. Método - Em estudo longitudinal retrospectivo analisaram-se 62 prontuários de cirróticos operados pela técnica de Teixeira-Warren na Santa Casa de São Paulo. Foram submetidos a avaliação quanto ao índice de congestão portal pré-operatório 36 doentes, e 58 quanto à classe Child-Pugh. Os doentes foram divididos em grupos - com recidiva e ausência de recidiva hemorrágica - sendo analisada a diferença estatística quanto aos valores do índice e Child-Pugh pré-operatórios, relacionando-os à recidiva hemorrágica pós-operatória. Resultados - Dos doentes que apresentaram recidiva hemorrágica, $69 \%$ eram Child B e possuíam índice de congestão portal médio de 0,09 . Já entre aqueles que não ressangraram, $62 \%$ eram Child A e o índice de congestão portal médio foi de 0,076. A diferença foi estatisticamente significante para a classe Child-Pugh, porém, o mesmo não ocorreu para o índice de congestão portal. Conclusão - O índice de congestão portal no pré-operatório não foi fator preditivo de recidiva hemorrágica em cirróticos submetidos a cirurgia de Teixeira-Warren. Doentes classificados como Child-Pugh B possuem maior chance de recidiva hemorrágica pós-derivação esplenorrenal distal em relação aos Child-Pugh A.
\end{abstract}

DESCRITORES - Insuficiência hepática. Cirrose hepática. Derivação esplenorenal cirúrgica. Hipertensão portal.

\section{INTRODUÇÃO}

A cirurgia de Teixeira-Warren ${ }^{(34)}$ é uma derivação venosa portal seletiva, pela anastomose da veia esplênica à veia renal esquerda, associada à ligadura da veia gástrica esquerda e desconexão esplenopancreática, não se manipulando a região do hilo hepático. Diminui a pressão venosa do plexo esôfago-cardiotuberositário e, por conseqüência, os episódios de sangramento varicoso. Além disto, evita a completa fuga de fluxo sangüíneo da veia porta, preservando parcialmente a perfusão e portanto a função hepática, graças à manutenção da hipertensão venosa no território intestinal, reduzindo a incidência de encefalopatia hepática ${ }^{(6,33,34)}$.

Quando a intervenção cirúrgica não é possível ou em doentes que estão à espera de transplante hepático, uma alternativa é a realização da derivação portossistêmica intra-hepática (TIPS). Inicialmente, quando os enxertos auto-expansíveis não eram revestidos, tinha-se alto índice de obstrução após 6 a 12 meses de uso, devendo ser trocados, implicando em aumento da morbimortalidade, principalmente por recidiva hemorrágica ${ }^{(1)}$. Hoje em dia, com a melhora tecnológica e o surgimento de enxertos revestidos por politetra-fluoroetileno, a patência do TIPS melhorou muito, variando de $80 \%$ a $84 \%$ em 1 ano e chegando a $75 \%$ no final de 2 anos, porém sem melhora na taxa de mortalidade, própria da cirrose $e^{(1,4,7,12,30,32)}$. Infelizmente, nem sempre esse tratamento está disponível em todos os serviços, principalmente no sistema público, devido ao seu elevado custo.

O índice de congestão portal (ICP) é calculado baseandose em dados fornecidos pela ultra-sonografia Doppler (USDoppler) e encontra-se elevado em doentes com hipertensão portal devido ao acréscimo da resistência ao fluxo portal ${ }^{(20)}$,

Trabalho realizado no Grupo de Fígado e Hipertensão Portal do Departamento de Cirurgia da Faculdade de Ciências Médicas da Santa Casa de São Paulo.

'Santa Casa de São Paulo, SP; ${ }^{2}$ Pontifícia Universidade Católica do Paraná, Curitiba, PR.

Correspondência: Dr. Fabio Gonçalves Ferreira - Rua Apinagés, 1060 - apt. 93 - Perdizes - 05017-000 - São Paulo, SP. E-mail drfabioferreira@uol.com.br 
podendo até ter valor na avaliação pré-operatória como preditor de trombose portal em esquistossomóticos operados ${ }^{(10)}$.

O objetivo deste estudo foi verificar se o grau de insuficiência hepática e o ICP pré-operatórios são fatores preditivos de recidiva hemorrágica em doentes cirróticos submetidos a cirurgia de Teixeira-Warren.

\section{MÉTODO}

Realizou-se estudo longitudinal retrospectivo através da revisão dos prontuários dos indivíduos submetidos a cirurgia de Teixeira-Warren pelo Grupo de Fígado e Hipertensão Portal da Santa Casa de São Paulo, de janeiro de 1983 a setembro de 2005.

O estudo foi aprovado pelo Comitê de Ética e Pesquisa em Seres Humanos da Irmandade Santa Casa de Misericórdia de São Paulo.

Foram operados neste período 62 doentes com diagnóstico de hipertensão portal por cirrose de causas viral, alcoólica, mista (cirrose + esquistossomose), não definida, medicamentosa ou fibrose hepática congênita classes Child A e B. A média de idade foi de 47 anos, com predomínio do sexo masculino (77\%).

Dos 62 pacientes operados, 4 foram excluídos para a análise de recidiva hemorrágica relacionada à classe Child-Pugh, pois evoluíram com trombose da anastomose esplenorrenal. Os 58 pacientes restantes foram divididos em dois grupos: grupo 1 (recidiva hemorrágica) e grupo 2 (ausência de recidiva). Nesses grupos os doentes foram ordenados de acordo com a classificação de Child-Pugh ${ }^{(24)}$ (A e B) e determinou-se a percentagem de pacientes em cada classe que apresentou recidiva hemorrágica.

Os pacientes cuja soma na pontuação de Child-Pugh foi igual a 5 ou 6, foram classificados como classe Child-Pugh A, enquanto que os classe B apresentaram pontuação igual a 7, 8 ou 9 (Tabela 1$)^{(24)}$.

Para a análise do ICP foram excluídos 24 doentes que não possuíam todos os parâmetros ao US-Doppler necessários para seu cálculo. Além desses, foram também excluídos 2 doentes que evoluíram com trombose da anastomose, restando 36 doentes para análise do ICP. Estes últimos foram divididos em dois grupos: grupo 3 (recidiva hemorrágica) e grupo 4 (ausência de recidiva).

Todos os 36 pacientes analisados quanto ao ICP foram avaliados no pré-operatório com US-Doppler no Serviço de Diagnóstico por Imagem da Santa Casa de São Paulo. Utilizou-se um aparelho de ultra-sonografia bidimensional de tempo real, acoplado ao Doppler de onda pulsátil da marca Toshiba ${ }^{\circledR}$, modelo Sonolayer SSH I40A, Japão, com transdutor de 3,75 MHz. Analisaram-se os seguintes parâmetros ultra-sonográficos: diâmetro da veia porta (DVP), área elíptica da veia porta $\left(\mathrm{cm}^{2}\right)$ calculada pela fórmula ${ }^{(31)} \mathrm{AVP}=(\mathrm{DVP})^{2} \mathrm{X} \pi / 4$, velocidade de fluxo na veia porta $(\mathrm{cm} / \mathrm{s})$ e fluxo de sangue na veia porta $(\mathrm{mL} / \mathrm{min})$ obtido com a fórmula ${ }^{(4)} \mathrm{F}=$ velocidade de fluxo x AVP $\mathrm{x} 60$. Com estes dados, calculou-se o índice de congestão portal $(\mathrm{cm} / \mathrm{s})$ pela fórmula proposta por MORIYASU et al. ${ }^{(20)} \mathrm{ICP}=$ $\mathrm{AVP} /$ velocidade de fluxo sangüíneo na veia porta.
Dos valores analisados entre os grupos 3 e 4 obteve-se a média e o desvio-padrão, submetendo-os a análise estatística. Utilizou-se o teste $t$ de Student na comparação entre os grupos, considerando-se $P \leq 0,05$ como nível de rejeição da hipótese nula. O teste exato de Fisher foi usado para comparação dos grupos 1 e 2 em relação à classe Child-Pugh. Considerou-se $P \leq 0,05$ como nível de rejeição da hipótese nula.

\section{RESULTADOS}

Dos 62 doentes operados, 35 apresentaram recidiva hemorrágica (56\%). Estes foram acompanhados num período que variou de 2 meses a 10 anos (média de 3,2 anos).

Quanto à classificação de Child-Pugh, a percentagem de pacientes Child B foi maior no grupo 1 (69\%), enquanto que no grupo 2 foi de $38 \%$. A diferença foi estatisticamente significante (Tabela 2). Nos doentes operados Child-Pugh A, a recidiva hemorrágica foi de $39 \%$, enquanto que nos Child-Pugh B, aconteceu em $69 \%$ dos casos.

As médias dos valores do ICP, área da veia porta e o fluxo na veia porta foram maiores no grupo 3 (recidiva hemorrágica). No tocante à velocidade de fluxo na veia porta, essa foi maior no grupo 4 (ausência de recidiva). Em nenhum dos parâmetros a diferença foi estatisticamente significativa entre os grupos (Tabela 3).

TABELA 1. Classificação de Child-Pugh: parâmetros avaliados e pontuação correspondente

\begin{tabular}{lccc}
\hline & 1 & 2 & 3 \\
\hline Encefalopatia & Ausente & Graus 1 e 2 & Graus 3 e 4 \\
Ascite & Ausente & Leve/moderada & Tensa \\
Bilirrubinas & $<2$ & $2-3$ & $>3$ \\
Albumina & $>3,5$ & $2,8-3,5$ & $<2,8$ \\
Atividade/tempo de protrombina & $>70 \%$ & $50 \%-70 \%$ & $<50 \%$ \\
& $<4 \mathrm{seg}$ & $4-6 \mathrm{seg}$ & $>6 \mathrm{seg}$ \\
\hline
\end{tabular}

5-6: A; 7-9: B; 10-15: C

TABELA 2. Classificação de Child-Pugh nos grupos comparados: $1=$ recidiva hemorrágica e 2 = sem recidiva hemorrágica

\begin{tabular}{lccc}
\hline Child-Pugh & Grupo 1 & Grupo 2 & \multirow{2}{*}{$\boldsymbol{P}^{*}$} \\
\hline \multirow{2}{*}{$\mathrm{n}$} & $\mathrm{n}=32$ & 10 & 0,03 \\
& $(31 \%)$ & $(62 \%)$ & \\
\multirow{2}{*}{$\mathrm{B}$} & 22 & 10 & \\
& $(69 \%)$ & $(39 \%)$ & \\
\hline
\end{tabular}

*estatisticamente significante $(P \leq 0,05)$

TABELA 3. Médias e desvios-padrão dos parâmetros ao US-Doppler nos pacientes cirróticos submetidos a cirurgia de Teixeira-Warren $(n=36)$ : 3 = recidiva hemorrágica e $4=$ sem recidiva hemorrágica

\begin{tabular}{lccc}
\hline Parâmetro pré-operatório & $\begin{array}{c}\text { Grupo 3 } \\
(\mathrm{n}=17)\end{array}$ & $\begin{array}{c}\text { Grupo 4 } \\
(\mathrm{n}=19)\end{array}$ & $\boldsymbol{P}^{*}$ \\
\hline Área da veia porta $\left(\mathrm{cm}^{2}\right)$ & $1,33 \pm 0,53$ & $1,23 \pm 0,55$ & 0,56 \\
Velocidade de fluxo na veia porta $(\mathrm{cm} / \mathrm{s})$ & $17,65 \pm 7,04$ & $18,10 \pm 6,14$ & 0,84 \\
Fluxo da veia porta $(\mathrm{ml} / \mathrm{min})$ & $1398,96 \pm 780,31$ & $1376,29 \pm 874,95$ & 0,94 \\
Índice de congestão portal $(\mathrm{cm} / \mathrm{s})$ & $0,090 \pm 0,063$ & $0,076 \pm 0,051$ & 0,47 \\
\hline * nenhum dos valores foi significante estatisticamente & & &
\end{tabular}




\section{DISCUSSÃO}

Além do calibre da veia esplênica, que deve ser próximo a $1,0 \mathrm{~cm}$, o principal fator, e que sempre foi preocupação na indicação da cirurgia de Teixeira-Warren, foi a reserva funcional hepática. Até pouco tempo, operavam-se todos os cirróticos nas condições acima que apresentassem função hepática classificada como Child A e B.

Com o advento do transplante hepático como forma de tratamento definitivo para cirróticos com alteração da função hepática, o manuseio dos doentes com sangramento por varizes esôfago-gástricas sofreu algumas alterações. Nos cirróticos com função hepática preservada (Child $\mathrm{A})$, o tratamento clínico-endoscópico parece ser o mais adequado. Na falha deste tratamento, que na literatura varia de $30 \%$ a $71 \%$ dos $\operatorname{casos}^{(2,18 \text {, }}$ 23, 27), ainda se indica cirurgia de Teixeira-Warren.

Não se discute que o melhor tratamento para cirróticos com função hepática alterada (Child B ou C), após o controle do surto agudo de hemorragia varicosa, é o transplante hepático. Para esses doentes indica-se o tratamento clínico-endoscópico e posterior avaliação do grupo de transplante hepático. Caso venham a ser listados para transplante e apresentem recidiva hemorrágica caracterizada como falha do tratamento clínico-endoscópico (programa de escleroterapia em ambulatório e fármacos que reduzam a pressão portal de forma efetiva), indica-se a passagem do TIPS. Porém, como só recentemente esse tratamento foi adicionado à lista de procedimentos custeados pelo sistema público de saúde e somente com os enxertos não revestidos de poli-tetra-fluoroetileno, por algum tempo continuou-se a indicar a cirurgia de Teixeira-Warren para os cirróticos Child B que falhavam no tratamento clínico-endoscópico. Além disso, a fila para o transplante é bastante longa em nosso meio.

As complicações mais comuns da cirurgia de Teixeira-Warren são a recidiva hemorrágica, ascite, trombose da anastomose esplenorenal e encefalopatia hepática ${ }^{(6,19)}$.

Em estudo recente ${ }^{(12)}$, observou-se alto índice de recidiva hemorrágica após essa operação, mesmo com anastomose pérvia $^{(9)}$. Nesses casos, a derivação esplenorenal seletiva parece não manter a descompressão adequada do sistema portal, que poderia traduzir inadequada diferença de pressão entre os sistemas portal e cava inferior, culminando em recidiva hemorrágica. Ou ainda, a presença de número expressivo de doentes com pior reserva hepática nesta casuística e que após a cirurgia teriam sua função piorada. Não se pode deixar de lado também a própria evolução da hepatopatia que, com o passar do tempo, ocorre piora progressiva da função hepática, dependendo da sua causa.

Nesse mesmo estudo ${ }^{(9)}$, em que se revisou retrospectivamente a recidiva hemorrágica nos pacientes Child $\mathrm{A}$ e $\mathrm{B}$ operados pela técnica de Teixeira-Warren, observou-se que a percentagem de ressangramento $(56 \%)$ foi mais acentuada do que na literatura $(3,8 \%-14 \%)^{(13,15,16,17,21,28)}$. Atribui-se esse índice ao elevado número de doentes Child $\mathrm{B}$ e de cirrose por álcool. Além disso, muitos doentes foram operados mais precocemente, logo após a compensação do episódio hemorrágico, na mesma internação.

Neste estudo conseguiu-se mostrar que os cirróticos com menor reserva hepática (Child B), apresentaram índice de recidiva hemorrágica por varizes esôfago-gástricas significantemente maior que nos doentes Child A após a cirurgia de Teixeira-Warren.

Para WONG et al. ${ }^{(35)}$, a derivação esplenorenal distal é um bom método para tratamento dos doentes Child $\mathrm{A}$ e $\mathrm{B}$ que não possuam fácil acesso ao TIPS, aos programas de escleroterapia e ao transplante hepático. De 34 pacientes analisados (16 Child A e 18 Child C/B), houve apenas 1 recidiva hemorrágica. Em trabalho de revisão sobre o tratamento cirúrgico da hipertensão portal em cirróticos, SELZNER et al. ${ }^{(26)}$ estabeleceram um algoritmo para pacientes com sangramento recurrente por varizes esofágicas: pacientes Child Ce Child B com ascite devem ser tratados com TIPS como "ponte" para o transplante hepático. Já nos Child A e B sem ascite, o melhor tratamento seria a derivação esplenorenal distal. $\mathrm{Na}$ impossibilidade ou falha desta técnica, o procedimento modificado de SUGIURA e FUTAGAWA ${ }^{(29)}$ poderia ser considerado.

Segundo CORSON et al. ${ }^{(5)}$, com a popularização dos procedimentos endovasculares (TIPS), o tratamento com descompressão portal cirúrgica em cirróticos ficou mais restrito aos casos em que houve falha dos métodos não-cirúrgicos em doentes com função hepática preservada. Sabe-se, porém, que a espera pelo transplante hepático é longa em nosso meio e que o TIPS custeado pelo sistema público apresenta perviedade em torno de 1 ano, havendo muitas vezes recidiva hemorrágica por trombose do enxerto não revestido de poli-tetra-fluoroetileno $(\text { PTFE })^{(18)}$. Em estudo caso-controle, HELTON et al. ${ }^{(14)}$ concluíram que em doentes Child-Pugh A e B os shunts cirúrgicos são mais efetivos, mais duráveis e mais baratos que o TIPS. Apesar disso, pacientes com pontuação Child-Pugh 12 e MELD 25 apresentam maior mortalidade e complicações pelo TIPS, do que doentes com melhor reserva hepática tratados pela mesma modalidade e devem ser analisados com critério ${ }^{(8)}$.

Mais recentemente, têm-se obtido bons resultados com o TIPS revestido com PTFE expansível (Viatorr). Segundo HAUSEGGER et al. ${ }^{(12)}$, que acompanhou pacientes de todas as classes hepáticas funcionais tratados com este dispositivo, os índices de patência da prótese são de $80 \%$ em 12 meses. Obtiveram valor bastante reduzido de recidiva hemorrágica $(3,7 \%)$, acompanhando seus pacientes, em média, por 18 meses.

De acordo com SCHIEDERMEIER ${ }^{(25)}$, o ICP eleva-se com a resistência ao fluxo portal, estando elevado em cirróticos, enquanto o fluxo portal está reduzido. Segundo MORIYASU et al. ${ }^{(20)}$, o ICP foi 2,5 vezes maior tanto em cirróticos quanto em doentes com hipertensão portal idiopática, em comparação com a população normal. Para eles, o ICP é mais sensível que a velocidade de fluxo, a área e o volume de fluxo na veia porta calculados pelo US-Doppler no diagnóstico de hipertensão portal. Os resultados do presente estudo demonstraram que a média dos parâmetros ultra-sonográficos pré-operatórios - ICP e área da veia porta - eram ligeiramente aumentados e a velocidade de fluxo na mesma reduzida nos doentes que apresentaram recidiva hemorrágica, quando comparados aos que não ressangraram. Estes resultados seguem o mesmo padrão daqueles apresentados por MORIYASU et al. ${ }^{(20)}$, no entanto, não houve significância estatística.

Obteve-se significado estatístico quanto aos valores do Child-Pugh pré-operatórios, o que mostrou ser este índice preditivo de recidiva hemorrágica em pacientes submetidos 
a derivação espleno-renal distal. No estudo de FLAMANT et al. ${ }^{(11)}$, os fatores preditivos de ressangramento foram o número de episódios prévios de hemorragia digestiva alta e a idade. Já quanto à classificação de Child-Pugh como fator preditivo, OROZCO et al. ${ }^{(23)}$ verificaram $5 \%$ de recidiva hemorrágica em doentes Child A contra 16\% nos Child B e C, dado este que foi significante.

Diversos trabalhos se propuseram a definir os fatores preditivos de recidiva hemorrágica nesta cirurgia, porém os resultados são conflitantes e novos estudos devem ser realizados na busca de uma resposta definitiva de índices prognósticos, sem lançar mão de métodos invasivos, que podem ser danosos ao paciente ${ }^{(9,22)}$.

Acredita-se que com a aparente melhora nos índices de patência dos novos enxertos revestidos de PTFE, a cirurgia de
Teixeira-Warren, antes de indicação restrita no grupo de Fígado e Hipertensão Portal da Faculdade de Ciências Médicas da Santa Casa de São Paulo, poderá ser ainda menos indicada no tratamento dos cirróticos com hemorragia varicosa.

\section{CONCLUSÃO}

O índice de congestão portal no pré-operatório não foi fator preditivo de recidiva hemorrágica em cirróticos com falha do tratamento clínico endoscópico submetidos a cirurgia de Teixeira-Warren.

Cirróticos Child-Pugh B possuem maior chance de recidiva hemorrágica pós-derivação espleno-renal distal em relação aos Child-Pugh A.

Ferreira FG, Duda JR, Olandoski M, De Cápua Jr. A. Role of liver function and portal vein congestion index on rebleeding in cirrhotics after distal splenorenal shunt. Arq Gastroenterol. 2007;44(2):123-7.

ABSTRACT - Background - Bleeding from esophagogastric varices is the worst and most lethal complication of cirrhotic portal hypertension. Distal splenorenal shunt (Warren's surgery) is used in the therapeutic of this patients, Child A and B, with rebleeding after clinical endoscopic therapy. The portal vein congestion index is elevated in cirrhotic portal hypertension and could predict rebleeding after Warren's surgery in these patients. Aim - To verify if the portal vein congestion index or liver function (Child-Pugh) at preoperative are predictive factors of rebleeding after Warren's surgery. Methods - Sixty-two cirrhotic patients were submitted to Warren's surgery at "Santa Casa" Medical School and Hospital - Liver and Portal Hypertension Unit, São Paulo, SP, Brazil. Fiftyeight were analyzed for Child-Pugh class and 36 for portal vein congestion index, divided in two groups: with or without rebleeding and statistical analysis was performed. Results - In the rebleeding group, $69 \%$ were Child B, with portal vein congestion index $=0.09$. The group without rebleeding show us $62 \%$ patients Child A with portal vein congestion index $=0.076$. The difference was significant for Child-Pugh class but not to portal vein congestion index. Conclusion - Portal vein congestion index was not predictive of rebleeding after Warren's surgery, but cirrhotics Child B have more chance to rebleed after this surgery than Child A.

HEADINGS - Hepatic insufficiency. Liver cirrhosis. Splenorenal shunt, surgical. Hypertension, portal. 


\section{REFERÊNCIAS}

1. Amarapurkar DN, Punamiya S, Patel ND. An experience with covered transjugular intrahepatic portosystemic shunt for refractory ascites from western India. Ann Hepatol. 2006;5:103-8.

2. Bosch J, Garcia-Pagan JC. Prevention of variceal rebleeding. Lancet. 2003;361:952-4.

3. Brown HS, Halliwel M, Qamar M, Read AE, Evans JM, Wells PN. Measurement of normal portal venous blood flow by Doppler ultrasound. Gut. 1989;30:503-9.

4. Charon JP, Alaeddin FH, Pimpalwar SA, Fay DM, Olliff SP, Jackson RW, Edwards $\mathrm{RD}$, Robertson IR, Rose JD, Moss JG. Results of a retrospective multicenter trial of the Viatorr expanded polytetrafluoroethylene-covered stent-graft for transjugular intrahepatic portosystemic shunt creation. J Vasc Interv Radiol. 2004;15:1219-30.

5. Corson JD, Williamson RCN, editors. Surgery. London: Mosby-Year Book; 2001.

6. Cury AA, Marx-Filho C, Carvalho NN. Hipertensão portal: anastomose espleno-renal distal seletiva. Rev Col Bras Cir. 1983;10:126-34.

7. Fanelli F, Salvatori FM, Corona M, Bruni A, Pucci A, Boatta E, Dominelli V, Conchiglia A, Passariello R. Stent graft in TIPS: technical and procedural aspects. Radiol Med. (Torino) 2006;111:709-23.

8. Ferral H, Patel NH. Selection criteria for patients undergoing transjugular intrahepatic portosystemic shunt procedures: current status. J Vasc Interv Radiol. 2005; $16: 449-55$

9. Ferreira FG, Saliture-Neto FT, Santos MF, Assef JC, Szutan LA, De Cápua Jr A. Fatores preditivos de recidiva hemorrágica em cirróticos submetidos a cirurgia de Teixeira-Warren. Rev Assoc Med Bras. 2005;51:261-4.

10. Ferreira FG, Chin EWK, Santos MF, Carvalho DLM, De Cápua Jr A. Índice de congestão portal e a ocorrência de trombose portal pós DAPE. Rev Assoc Med Bras. 2005;51:233-6.

11. Flamant Y, Collard JM, Sicoli F, Hay JM, Maillard JN. L'anastomose spléno-rénale distale dans le traitement des hémorragies digestives de l'hypertension portale. Ann Chir 1991;45:340-3.

12. Hausegger KA, Karnel F, Georgieva B, Tauss J, Portugaller H, Deutschmann H, Berghold A. Transjugular intrahepatic portosystemic shunt creation with the Viatorr Expanded Polytetrafluoroethylene-Covered Stent-Graft. J Vasc Interv Radiol. 2004;15:239-48.

13. He B, Zang Z, Zhou Q. Clinical application of splenorenal shunt. Zhonghua Wai Ke Za Zhi. 1996;34:416-20.

14. Helton WS, Maves R, Wicks K, Johansen K. Transjugular intrahepatic portasystemic shunt vs. surgical shunt in good-risk cirrhotic patients: a case-control comparison. Arch Surg. 2001;136:17-20.

15. Henderson JM, Nagel A, Curtas S, Geisinger M, Barnes D. Surgical shunts and TIPS for variceal decompression in the 1990s. Surgery. 2000;128:540-7.

16. Huang Y, Wang W, Wang J, Bai C. Surgical treatment of portal hypertension: 45 year experience. Zhonghua Wai Ke Za Zhi. 2000;38:85-8.

17. Jenkins RL, Gedaly R, Pomposelli JJ, Pomfret EA, Gordon F, Lewis WD. Distal splenorenal shunt: role, indications, and utility in the era of liver transplantation. Arch Surg. 1999;134:416-20.

18. Kalil AN, Coelho JCU, Adam R. Hipertensão portal. In: Coelho JCU, editor. Aparelho digestivo. Clínica e cirurgia. São Paulo: Atheneu; 2005. p.1274-93.

19. Khaitiyar JS, Luthra SK, Prasad N, Ratnakar N, Daruwala DK. Transjugular intrahepatic portosystemic shunt versus distal splenorenal shunt: a comparative study. Hepatogastroenterology. 2000;47:492-7.
20. Moriyasu F, Nishida O, Ban N, Nakamura T, Sakai M, Miyake T, Uchino H. "Congestion Index" of the portal vein. AJR Am J Roentgenol. 1986;146:735-9.

21. Nagasue N, Kohno H, Ogawa Y, Yukaya H, Tamada R, Sasaki Y, Chang YC, Nakamura T. Appraisal of distal splenorenal shunt in the treatment of esophageal varices: an analysis of prophylactic, emergency, and elective shunts. World J Surg. 1989;13:92-9.

22. Orozco H, Mercado MA. The evolution of portal hypertension surgery: lessons from 1000 operations and 50 years' experience. Arch Surg. 2000;135:1389-93.

23. Orozco H, Mercado MA, Chan C, Guillen-Navarro E, Lopez-Martinez LM. A comparative study of the elective treatment of variceal hemorrhage with beta-blockers, transendoscopic sclerotherapy and surgery: a prospective, controlled and randomized trial during 10 years. Ann Surg. 2000;232:216-9.

24. Pugh RN, Murray-Lyon IM, Dawson JL, Pietroni MC, Williams R. Transection of the oesophagus for bleeding oesophageal varices. Br J Surg. 1973;60:646-9.

25. Schiedermaier P. Splanchnic hemodynamics: cirrhotic versus non-cirrhotic porta hypertension. J Gastroenterol Hepatol. 2004;19 Suppl 7:s150-s4

26. Selzner M, Tuttle-Newhall JE, Dahm F, Suhocki P, Clavien PA. Current indication of a modified Sugiura procedure in the management of variceal bleeding. J Am Coll Surg. 2001;193:166-73.

27. Singh P, Pooran N, Indaram A, Bank S. Combined ligation and sclerotherapy versus ligation alone for secondary prophylaxis of esophageal variceal bleeding: a metaanalysis. Am J Gastroenterol. 2002;97:623-9.

28. Spina GP, Galeotti F, Opocher E, Santambrogio R, Cucchiaro G, Lopez C, Pezzuol G. Selective distal splenorenal shunt versus side-to-side porto-caval shunt. Clinical results of a prospective controlled study. Am J Surg. 1988;155:564-71.

29. Sugiura M, Futagawa S. A new technique for treating esophageal varices. J Thorac Cardiovasc Surg. 1973;66:677-85.

30. Tripathi D, Ferguson J, Barkell H, Macbeth K, Ireland H, Redhead DN, Hayes PC. Improved clinical outcome with transjugular intrahepatic portosystemic stent-shunt utilizing polytetrafluoroethylene-covered stents. Eur J Gastroenterol Hepatol. 2006;18:225-32

31. Verozzo DCP. Avaliação hepática e hemodinâmica portal com Doppler duplex na esquistossomose mansônica [dissertação]. São Paulo: Faculdade de Medicina da Universidade de São Paulo; 1992.

32. Vignali C, Bargellini I, Grosso M, Passalacqua G, Maglione F, Pedrazzini F, Filauri P, Niola R, Cioni R, Petruzzi P. TIPS with expanded polytetrafluoroethylene-covered stent: results of an Italian multicenter study. AJR Am J Roentgenol. 2005;185:472-80

33. Warren WD, Millikan WJ Jr, Henderson JM. Splenopancreatic disconnection. Ann Surg. 1986; 204:346-55.

34. Warren WD, Zeppa R, Fomon JJ. Selective trans-splenic decompression of gastroesophageal varices by distal splenorenal shunt. Ann Surg. 1967;166:437-55.

35. Wong LL, Lorenzo C, Limm M, Wong LM. Splenorenal shunt: an ideal procedure in the pacific. Arch Surg. 2002;137:1125-9. 